\title{
Entre la comprensión y la predicción del funcionamiento de catalizadores sólidos ${ }^{\diamond}$
}

\section{Between understanding and predicting the performance of solid catalysts}

\author{
Juan C. Fierro-Gonzalez*
}

\begin{abstract}
Solid catalysts discovery is generally done by using methodologies that involve trialand-error. In the best cases, this strategy is supported by information obtained from characterization techniques. However, the structural complexity of typical solid catalysts complicates the interpretation of data, and there are no detailed and rigorous descriptions of the way in which solid catalysts function. Furthermore, the trial-and-error strategy does not allow to predict whether the synthesized materials will be the best possible for a specific reaction. In contrast, the rational design of solid catalysts is an alternative to the trial-and-error that could serve, at least in principle, to predict the structure that is required for a material to be catalytically active for a specific chemical reaction. This strategy, however, requires fundamental knowledge of the materials (i.e., specific and rigorous details of the nature of the active sites), which is a challenging task. In this work, the strategies that are typically used to study solid catalysts are contrasted, placing special emphasis on the possibility of designing materials. What it means to understand catalyst performance at a fundamental level is discussed, and a critical analysis is made of potential opportunities that design offers to address some relevant issues in catalyst study today.
\end{abstract}

KEYWORDS: catalysts design, trial-and-error, catalytic cycles.

RESUMEN: El descubrimiento de catalizadores sólidos generalmente se realiza a través de una metodología que incorpora elementos de prueba y error. En el mejor de los casos, esta estrategia se apoya en información obtenida de técnicas de caracterización. Sin embargo, como la estructura de los catalizadores sólidos es generalmente complicada, dicha información es insuficiente para tener una descripción precisa y rigurosa del modo en que funcionan los catalizadores. Además, la estrategia tampoco permite establecer si los catalizadores encontrados serán los mejores posibles. Como contraparte de la prueba y error está el diseño de catalizadores, con el cual se pretende desarrollar materiales a partir de su comprensión a nivel fundamental (i.e., detalles específicos y rigurosos de la naturaleza del sitio activo). En principio, el diseño permitiría predecir la estructura necesaria en un material para que este sea catalíticamente activo. En este trabajo se contrastan las estrategias que típicamente se emplean para estudiar catalizadores sólidos, poniendo énfasis especial en la posibilidad de diseñar materiales. Se discute qué significa comprender el funcionamiento de los catalizadores a nivel fundamental y se hace un

Recibido: 2 de mayo de 2020.

Aceptado: 14 de junio de 2020.

${ }^{\bullet}$ Agradecimientos. El autor agradece al Tecnológico Nacional de México por el financiamiento a través de la convocatoria de Apoyo a la Investigación Científica y Tecnológica en los Programas Educativos de los Institutos Tecnológicos Federales y Centros (proyectos 6404.18-P y 7875.20-P).

* Tecnológico Nacional de México en Celaya, Departamento de Ingeniería Química.

Correo electrónico: jcfierro@iqcelaya.itc.mx 
análisis crítico de posibles oportunidades que ofrece el diseño para abordar algunos problemas relevantes en el estudio de catalizadores en la actualidad.

PALABRAS CLAVE: diseño de catalizadores, prueba y error, ciclos catalíticos.

\section{Importancia de los catalizadores sólidos}

Los catalizadores son definidos por la Unión Internacional de Química Pura y Aplicada (IUPAC, por sus siglas en inglés) "como sustancias que aumentan la velocidad de una reacción química sin modificar el cambio en energía libre de Gibbs" (McNaught y Wilkinson, 1997). Cuando el catalizador se encuentra en la misma fase que los reactivos y los productos, se dice que ocurre catálisis homogénea. En cambio, cuando el catalizador está en una fase distinta a los reactivos y los productos se habla de catálisis heterogénea. En este último caso el catalizador es normalmente un sólido y su recuperación del medio de reacción es mucho más sencilla (también más barata) que en los procesos homogéneos. Existen además otras ventajas de los procesos heterogéneos, como su facilidad para realizarlos de manera continua y la posibilidad de llevarlos a cabo a altas temperaturas (sin el límite que imponen los solventes en los procesos homogéneos). Estas son algunas razones por las que los procesos heterogéneos son más utilizados a nivel industrial que los procesos de catálisis homogénea. Existen diversos tipos de catalizadores sólidos, entre los que se encuentran metales puros - casi siempre de transición- (Liu et al., 2013), óxidos metálicos (Hamada, 1994) y metales soportados (Bond, 1991) (casi siempre en zeolitas o en óxidos metálicos de alta área superficial). Generalmente, se considera que es la estructura a nivel nanométrico de la superficie de estos materiales la que les confiere sus propiedades como catalizadores (Goodman, 2008; Gates, 1995).

Los catalizadores sólidos son de gran importancia, pues se utilizan en procesos cruciales para la civilización moderna. Estos materiales se usan, por ejemplo, en varias etapas de la producción de combustibles (Corma et al., 2017; Primo y García, 2014) como la gasolina y el diésel, pero también se emplean en reacciones relevantes para disminuir las emisiones a la atmósfera que se generan cuando estos combustibles se utilizan en automóviles o en la industria (por caso, en la reducción de óxidos de nitrógeno) (Burch et al., 2002; He y Yu, 2005). Por su parte, la síntesis de metanol (De María et al., 2013) —compuesto esencial para producir una gran variedad de productos químicos-, también utiliza catalizadores sólidos, por citar solo algunos ejemplos.

Desarrollar catalizadores sólidos representa retos científicos y tecnológicos sustanciales por varias razones. Una de ellas es la notable dificultad que existe para conocer a priori la estructura superficial que se requiere para que un material funcione como catalizador en una reacción específica. Más aún, incluso en aquellos casos en los que se tiene suficiente conocimiento sobre los sitios necesarios para tener actividad catalítica, es complicado sintetizar materiales que únicamente contengan esos sitios. Normalmente, los 
métodos de síntesis de catalizadores sólidos dan lugar a superficies estructuralmente complicadas y poco uniformes, por lo que es difícil establecer con claridad dónde y cómo ocurren en realidad las reacciones catalíticas. Por ejemplo, en el caso de catalizadores de metales soportados, los materiales contienen partículas del metal de varios tamaños y formas que se encuentran distribuidas de manera prácticamente aleatoria sobre la superficie de un soporte poroso cuya superficie es también complicada. En la figura 1 se muestra una representación esquematizada de un catalizador de un metal soportado, donde puede verse la complejidad estructural y poca uniformidad superficial de estos materiales. Se compara con el caso de un catalizador de un complejo organometálico en solución (que típicamente se usaría en procesos homogéneos), en donde se tienen moléculas discretas del catalizador estabilizadas por un solvente.

Las dificultades enunciadas anteriormente para los catalizadores sólidos hacen que en la mayoría de los casos, el desarrollo de estos materiales dependa de estrategias en las que se tienen que sintetizar y probar múltiples catalizadores hasta encontrar aquel que parezca ser el mejor para una reacción en particular (Corma, 2016; Thomas, 1999; Corma et al., 2010). Puede decirse que el desarrollo de catalizadores es un proceso iterativo en el que la mejora de los materiales es paulatina y se dificulta en la medida en que la estructura de los catalizadores se vuelve más compleja. Existen, sin embargo, ejemplos en los que el desarrollo de los catalizadores se consiguió partiendo

Figura 1. Representaciones esquematizadas comparando un catalizador en solución y un catalizador de un metal soportado.

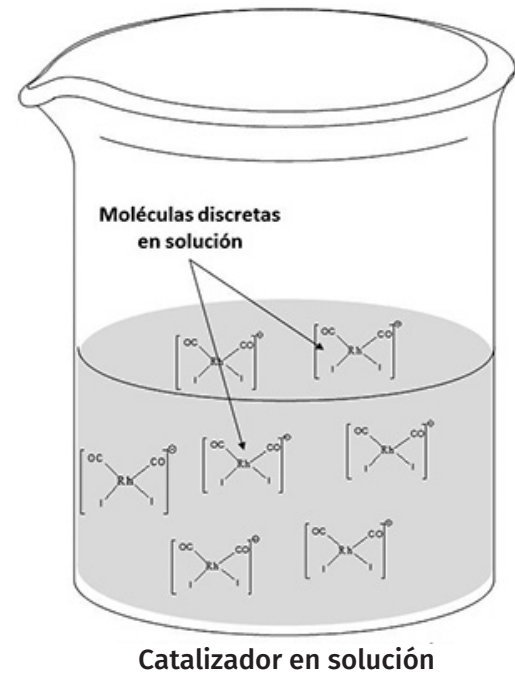

Partículas de diversos tamaños y formas sobre un soporte poroso

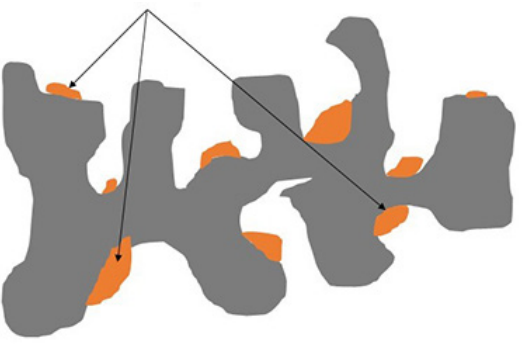

Fuente: Elaboración del autor.

\section{Catalizador sólido (metal soportado)}


de cierta comprensión a nivel fundamental de los sitios que estos deberían contener para funcionar. Estos ejemplos (algunos de ellos discutidos en este artículo) sugieren que en algunos casos es posible diseñar catalizadores sólidos, aunque sea a un nivel básico.

En este trabajo se contrastan las estrategias que típicamente se emplean para estudiar catalizadores sólidos, poniendo énfasis especial en la posibilidad de diseñar materiales. Se discute qué significa comprender el funcionamiento de los catalizadores a nivel fundamental y se hace un análisis crítico de posibles oportunidades que ofrece el diseño para abordar algunos problemas relevantes en el estudio de catalizadores en la actualidad.

\section{La comprensión como base del diseño de catalizadores sólidos}

Generalmente, la resolución de un problema puede realizarse mediante múltiples estrategias. Una de ellas es prueba y error, método heurístico en el que se ensayan posibles soluciones y se verifica su funcionamiento. En su extremo, esta estrategia no aspira a comprender el motivo por el que determinada solución funciona, sino que simplemente se orienta a resolver problemas específicos. Más aún, la solución obtenida no necesariamente será la óptima, por lo que esta estrategia puede seguirse aplicando indefinidamente sin tener una idea clara de si el óptimo ha sido alcanzado. Esto último está íntimamente ligado con el hecho de que la prueba y error (como método de solución) no pretende ni requiere conocer el origen del problema de manera profunda. Así pues, si se desconoce qué causa el problema será poco probable que se comprendan los alcances y limitaciones de la solución propuesta.

La prueba y error es tal vez la estrategia más utilizada en la investigación y desarrollo de catalizadores sólidos (Corma, 2016; Thomas, 1999; Corma et al., 2010). Desde el punto de vista de investigación, típicamente se parte de un problema que puede plantearse con una pregunta tan general como la siguiente: ¿qué catalizador funcionará para la reacción $\mathrm{X}$ ?

$\mathrm{Si}$ la reacción $\mathrm{X}$ ha sido estudiada antes (como comúnmente ocurre, a menos que el investigador sea realmente revolucionario y haya encontrado una reacción que nadie jamás imaginó antes), la pregunta se vuelve ligeramente más específica: ¿qué catalizador funcionará mejor para la reacción $\mathrm{X}$ ?

Esa ligera modificación a la pregunta coloca al investigador ante una situación interesante. Porque buscar un mejor catalizador se traduce en una comparación inevitable: ¿cómo es el mejor catalizador que ha sido descubierto hasta el momento para la reacción X? En un primer nivel, ese "cómo" genérico está asociado a qué constituye ese catalizador, es decir, de qué está hecho. Si el catalizador, por ejemplo, consiste de un metal M soportado en un soporte $S$, entonces nuestro investigador hipotético podría optar por imaginar distintas combinaciones de metales y soportes. Podría entonces anunciar ante el comité de posgrado de su universidad que necesita un par 
de estudiantes de maestría (si se puede de doctorado, ¡mejor aún!) y pedirles a esos hipotéticos estudiantes que se pongan a preparar tantas combinaciones metal/soporte como puedan y después probar sus materiales como catalizadores en la reacción X. Si al final de ese ejercicio tienen éxito, posiblemente una de esas combinaciones metal/soporte será mejor que la última que se encontraba reportada. El investigador y sus estudiantes podrían entonces intentar publicar sus hallazgos, pero en ese momento muy probablemente descubrirían que estos, en el mejor de los casos, simplemente les permiten describir un catalizador que es mejor a uno pre-existente, pero no tendrían elementos para asegurar que ese nuevo catalizador es inmejorable. Tampoco serían capaces de explicar a sus pares por qué razón este nuevo catalizador es mejor que el anterior. Si acaso, podrían aventurarse a plantear alguna hipótesis al respecto, pero esta sería especulativa al no contar con suficiente evidencia que la respalde.

El ejemplo anterior es un caso extremo del uso de prueba y error para desarrollar catalizadores. Puede verse que aunque la estrategia podría ser útil sería poco informativa en cuanto a la explicación del funcionamiento de los catalizadores. Regresemos al ejemplo e imaginemos otro escenario. Hemos dicho antes que el investigador quiere un mejor catalizador para la reacción $\mathrm{X}$. Supongamos que ahora, en lugar de únicamente analizar de qué está hecho el catalizador, el investigador se cuestiona sobre la estructura superficial del material, pues es ahí donde ocurre la reacción. Entonces, probablemente el investigador hará uso de distintas técnicas de caracterización, como microscopía o algunas variedades de espectroscopía, para conocer detalles sobre el catalizador que quiere mejorar. Al final, podría decir que ese catalizador tiene partículas del metal $\mathrm{M}$ con cierto diámetro promedio. Podría también explicar que el soporte $S$ tiene tal o cual acidez superficial, y seguramente también conocería el área superficial del material. Con esa información podría pedir a sus estudiantes que preparen catalizadores en los que alguna o todas esas características se modifiquen. Ellos lo harían y al final probarían sus materiales. Si tuvieran éxito, probablemente habrían encontrado un catalizador que sería mejor al último que se había reportado. Nuevamente, en este escenario de nuestro ejemplo intentarían publicar sus hallazgos, pero también en este caso serían incapaces de asegurar que el nuevo catalizador es inmejorable. Tendrían cierta evidencia para tratar de explicar por qué razón el nuevo catalizador es mejor que el anterior, pero difícilmente podrían tener certeza de que esa fuera la única razón. Además, llegar a ese nuevo catalizador también habría requerido de la síntesis de una variedad de materiales hasta hallar algo mejor. Entonces, también en este nuevo escenario nuestros personajes utilizaron la prueba y error para desarrollar un mejor catalizador para la reacción $\mathrm{X}$, solo que en este caso las pruebas se hicieron con base en algunas ideas sobre la estructura del catalizador a un nivel más fundamental.

Podemos continuar imaginando escenarios cada vez más complicados para este mismo ejemplo. En cada uno de ellos, nuestro investigador tendría 
que conocer a mayor detalle el modo en que funciona el catalizador para poder hacer propuestas de materiales. Los catalizadores cada vez serían mejores, al igual que las ideas que intentarían explicar su funcionamiento. Eventualmente, se llegaría a un extremo en el que el investigador tendría certeza sobre el modo exacto en el que funciona el mejor catalizador reportado hasta el momento. Si eso ocurriera (aunque evidentemente se trata de un supuesto), podría proponer cómo debería ser el mejor catalizador para la reacción X. Ese catalizador hipotético sería, efectivamente, inmejorable. Si eso fuera posible, diríamos que el investigador habría hallado el modo de diseñar un catalizador y predecir su funcionamiento.

En términos de la solución de cualquier problema, el diseño es una alternativa que se contrapone a la prueba y error, y puede definirse como un proceso mediante el cual se anticipa de manera racional qué elementos deberá contener una solución para que esta efectivamente resuelva el problema. Etimológicamente, la palabra diseñar proviene del latín designare, que significa designar, marcar o dibujar. El prefijo de se refiere a separar o disociar, mientras que la raíz signa o signum se refiere a una señal, marca o insignia de algo. Así pues, designare significa elegir, singularizar o separar algún signo o insignia de un todo. De este modo, al diseñar se eligen o se designan los elementos que deben estar presentes en la solución. A diferencia de la prueba $y$ error, la posible solución que se propone en un diseño no es aleatoria, sino que es elegida a partir de signos, marcas o insignias que son atribuibles al problema que se planea resolver. Por ello, el diseño requiere tener una idea profunda sobre el origen del problema. En otras palabras, existe una relación íntima entre la comprensión del origen del problema y el diseño, a tal grado que si dicha comprensión es suficientemente amplia la solución propuesta a partir del diseño debe funcionar. En ese sentido, el diseño anticipa o predice la solución a los problemas cuando el origen de estos es bien comprendido. Una vez alcanzado ese grado de maduración en el diseño, el reto consiste en convertirlo en una realidad tangible y aplicable que efectivamente resuelva el problema en la práctica. Esto último será posible en la medida en que existan herramientas tecnológicas suficientes para convertir el diseño en realidad.

Volvamos a nuestro ejemplo: supongamos ahora que el investigador comprende a gran detalle cómo funciona el mejor catalizador que ha sido reportado hasta el momento para la reacción X. Ese nivel de comprensión le permite identificar deficiencias en ese catalizador y establecer cuáles signos, marcas o insignias de ese material deben ser mejoradas para obtener el mejor catalizador posible. Efectivamente, nuestro investigador hipotético ya se encuentra diseñando un catalizador. Si todo va bien, al final de ese ejercicio tendrá una idea precisa de la estructura superficial que debería tener el mejor catalizador para la reacción X. En ese momento, podría llamar a sus estudiantes y pedirles que preparen ese material para demostrar su funcionamiento. Teóricamente, bastaría con que los estudiantes prepararan únicamente esa muestra (indicada por el diseño) para tener el mejor catalizador 
posible para la reacción X. Ya no sería necesario probar entre una variedad de opciones. ¡Ya puedo imaginar los rostros atónitos de los estudiantes! Porque en ese momento, el reto sería precisamente preparar con exactitud el material que surgió del diseño. Eso, amigo lector, normalmente puede representar un problema monumental en el ámbito de la catálisis heterogénea, pues la síntesis de materiales con estructuras superficiales específicas no es trivial.

Hasta ahora he utilizado un caso hipotético para distinguir entre dos estrategias aparentemente divergentes en el estudio de catalizadores: la prueba y error, y el diseño. Me he valido de extremos de ambas estrategias para resaltar sus diferencias. Sin embargo, la práctica tiende a ser menos radical que la filosofía en la que se sustenta el trabajo de los investigadores. Así pues, es cada vez menos común encontrar trabajos en los que se haga prueba y error sin intentar comprender (aunque sea un poco) el sistema que se estudia. Del mismo modo, hasta el momento no hay reportes en los que los autores hayan realmente hecho un trabajo puro de diseño, sin recurrir a información de trabajo iterativo de preparación de múltiples materiales. En todo caso, el diseño de catalizadores puede entenderse como una especie de objetivo aspiracional que al ser alcanzado tendría una evidente ventaja para desarrollar catalizadores.

\section{El ciclo catalítico y el sitio activo: piedras angulares en la comprensión de catalizadores}

Hemos dicho que el diseño requiere comprensión del problema que se pretende resolver. ¿Qué significa exactamente comprender el funcionamiento de los catalizadores? En catálisis homogénea es común encontrar reportes en los que se describe el modo en que funcionan los catalizadores en términos de su participación en el mecanismo de la reacción. Como un catalizador (por definición) no se consume durante la reacción neta, debe participar como reactivo en alguna reacción elemental del mecanismo y ser recuperado como producto en otra etapa. Entonces, los mecanismos de las reacciones catalíticas pueden escribirse en forma de ciclos en los que se verifican el consumo y recuperación continua del catalizador. En la figura 2 se muestra el ciclo catalítico que se ha propuesto para la hidrogenación de alquenos en presencia del catalizador de Wilkinson (Osbom et al., 1996), un complejo de organorodio en el que el metal se encuentra coordinado a cloro y ligantes de fosfinas. Cada una de las etapas del ciclo puede explicarse en detalle utilizando fundamentos químicos. Al inicio, una de las fosfinas se disocia y se forma un complejo de 14 electrones que es altamente reactivo. Posteriormente, este complejo experimenta una adición oxidativa de hidrógeno, formándose un complejo de 16 electrones al que puede coordinarse la olefina. Se ha propuesto que después puede ocurrir una inserción de la olefina en uno de los enlaces $\mathrm{Rh}-\mathrm{H}$ para formar un grupo alquil. Finalmente, mediante eliminación reductiva se forma el alcano y se cierra el ciclo catalítico con la recuperación del complejo de 14 electrones. 
Figura 2. Ciclo catalítico de la hidrogenación de olefinas en presencia del catalizador de Wilkinson.

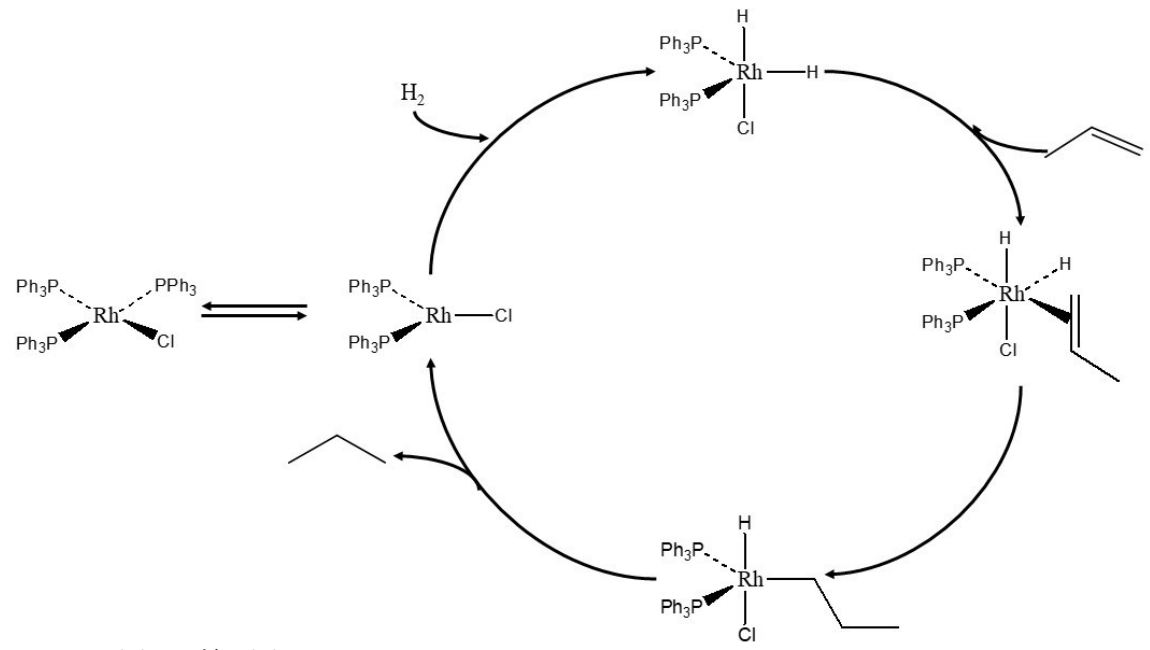

Fuente: Elaboración del autor.

La hidrogenación de alquenos usando el catalizador de Wilkinson es una de las reacciones de catálisis homogénea más estudiadas. Existe incluso suficiente evidencia espectroscópica para respaldar el mecanismo de la figura 2. Por ejemplo, mediante el uso de resonancia magnética nuclear se ha podido demostrar la adición oxidativa de $\mathrm{H}_{2}$ al complejo de 14 electrones (Brown et al., 1987). Además, cálculos ab initio han permitido estimar las energías relativas de diferentes estados de transición e intermediarios durante la reacción (Daniel et al., 1988). Más aún, los cálculos han demostrado que el paso limitante de la velocidad de reacción es la inserción de la olefina en el enlace $\mathrm{Rh}-\mathrm{H}$ (figura 1). Los cálculos son consistentes con expresiones cinéticas determinadas a partir de mediciones experimentales, por lo que el ciclo de la figura 1 cuenta con suficiente evidencia que lo respalda.

Existen muchos otros ejemplos similares al anterior, en los que el funcionamiento del catalizador es explicado a través de ciclos catalíticos que fueron propuestos a partir de evidencia física y cálculos teóricos. Algunos de ellos incluyen la carbonilación de metanol (Reppe et al., 1956; Paulik y Roth, 1968; Haynes et al., 2004) en presencia de catalizadores de Rh, Co e Ir, la producción de aldehídos a partir de reacciones de hidroformilación (Pruett y Smith, 1967) con catalizadores de Rh, y la oxidación de etileno a acetaldehído (Jira, 2009) en presencia de complejos de Pd, entre otros. Todos estos ejemplos tienen en común que los catalizadores utilizados son complejos organometálicos en solución, cuya química es relativamente bien conocida. Puede decirse que en estos casos los catalizadores tienen una uniformidad prácticamente absoluta, pues consisten de moléculas discretas de compuestos estabilizados por el solvente. Esta situación cambia considerablemente cuando se estudian 
catalizadores sólidos en los que las superficies son poco uniformes, pues estos contienen una variedad de sitios no equivalentes.

En teoría, los mismos principios que rigen los procesos de catálisis homogénea (ejemplificados por el ciclo catalítico de la figura 2) deberían explicar lo que ocurre en reacciones de catálisis heterogénea. Sin embargo, la complejidad estructural de los catalizadores sólidos y su poca uniformidad dificulta el estudio de mecanismos de reacción en presencia de estos materiales tanto por métodos experimentales como a través de química computacional. Por ello, el estudio de estos materiales normalmente se enfoca en intentar entender cuáles estructuras realmente participan en la reacción de interés. Los sitios superficiales que contienen esas estructuras son conocidos como sitios activos. Así, mientras que en reacciones de catálisis homogénea el ciclo catalítico se emplea con frecuencia para describir el funcionamiento de los catalizadores, en catálisis heterogénea las investigaciones se centran en elucidar la estructura de los sitios activos en los catalizadores sólidos.

Los complejos organometálicos en solución pueden describirse químicamente con cierta exactitud, es decir, se pueden dar detalles sobre ellos como el conteo de electrones, el estado de oxidación del metal, la identidad y naturaleza química de los ligantes coordinados al metal, entre otros. Es precisamente a partir de esa información que es posible proponer las reacciones químicas que constituyen el ciclo catalítico. En cambio, los sitios activos de los catalizadores sólidos no pueden describirse con la misma exactitud desde el punto de vista químico, sino que usualmente se representan en términos de variables estructurales menos rigurosas. Por ejemplo, si el catalizador es un metal soportado, es común que la representación del sitio activo se realice en términos del tamaño promedio de las partículas del metal, su estado de oxidación, el modo en que las partículas se encuentran ancladas al soporte, etc. Nuevamente, la complejidad estructural del catalizador sólido limita el grado de comprensión que de él puede tenerse. En la figura 1 se comparan esquemáticamente las estructuras de catalizadores en solución (usados en procesos homogéneos) y de un catalizador de un metal soportado (usado en un proceso heterogéneo). Nótese cómo en el catalizador en solución se trata de moléculas discretas de un complejo, cuyos enlaces químicos pueden describirse de manera rigurosa y exacta. Además, dichas moléculas se repetirán de manera uniforme en el solvente. En contraste, para representar el catalizador del metal soportado únicamente pueden esquematizarse partículas (con tamaños y formas promedio) de las que no pueden darse detalles específicos y rigurosos sobre la existencia y naturaleza de enlaces químicos que contienen. Más aún, no se puede establecer con certeza que dichas partículas sean realmente representativas de los sitios en los que ocurrirá la reacción química de interés (únicamente pueden hacerse propuestas basadas en cierta evidencia física).

De lo anterior se deriva que una estrategia útil para entender el funcionamiento de los catalizadores sólidos consiste en simplificar su estructura, pues entre más simple sea la estructura superficial de un catalizador, más 
simples serán los sitios activos y (en potencia) serán más fáciles de investigar y describir. Por lo tanto, existe una motivación por desarrollar métodos de síntesis para preparar catalizadores sólidos que sean lo suficientemente simples y uniformes en su estructura para ser considerados como análogos a catalizadores en solución. En principio, contar con sitios activos con estructuras cuasimoleculares podría permitir su caracterización a un mayor detalle y ganar de ese modo información relevante sobre el funcionamiento de los catalizadores sólidos. En las últimas décadas, diversos grupos de investigación (Argo et al., 2002; Yang et al., 2015; Fierro-González y Gates, 2004; Fierro-González et al., 2005; Fihri et al., 2011; Soulivong et al., 2008) han utilizado esta aproximación y han conseguido preparar catalizadores modelo con los que se puede adquirir conocimiento sobre su funcionamiento para algunas reacciones simples, como la hidrogenación de alquenos (Argo et al., 2002; Yang et al., 2015) la oxidación de monóxido de carbono (Fierro-González y Gates, 2004; Fierro-González et al., 2005) y acoplamientos carbonocarbono (Fihri et al., 2011; Soulivong et al., 2008), entre otras. Si bien la estrategia ha sido exitosa desde el punto de vista científico, los catalizadores modelo que se han obtenido normalmente no encuentran aplicación industrial, pues son poco estables y su simplicidad estructural depende de mantenerlos en atmósferas controladas y a temperaturas que normalmente son más bajas a las que se emplean en aplicaciones a gran escala.

Es importante no perder de vista que el interés fundamental debe ser el desarrollo de catalizadores con aplicaciones reales y no simplemente preparar materiales que justifiquen la demostración directa de conceptos químicos. En otras palabras, si un catalizador es simplificado de manera excesiva, el conocimiento que de él pudiera obtenerse sería irrelevante en el mundo práctico. Aunque existen algunos casos exitosos en los que catalizadores sólidos con estructuras considerablemente simples y generadas a partir de una estrategia parecida al diseño se utilizan en procesos industriales. Por ejemplo, catalizadores de cúmulos de platino en los poros de zeolita LTL son utilizados para el reformado selectivo de naftas para dar aromáticos (Jentoft et al., 1998) También, catalizadores de complejos de organorodio anclados a polivinilpirrolidona (PVP) se emplean para la carbonilación de metanol a nivel industrial (Minami et al., 1994). Este último ejemplo se discute a mayor detalle a continuación, haciendo énfasis en las ideas que dieron lugar al desarrollo del catalizador.

\section{Carbonilación de metanol}

La carbonilación de metanol es el principal proceso que se utiliza actualmente para obtener ácido acético a nivel industrial. El ácido acético es importante porque sirve como materia prima para la producción de una variedad de compuestos, como el acetato de vinilo y el monocloroacético (Haynes, 2010). En la década de los cincuenta, la empresa BASF desarrolló el primer proceso 
industrial de carbonilación de metanol, el cual ocurría en fase homogénea en presencia de un catalizador de $\mathrm{Co}(\mathrm{CO})^{4^{-}}$y yodometano (Reppe et al., 1956). Antes del proceso de BASF, las rutas principales para producir ácido acético eran la oxidación de etanol o de hidrocarburos. Estos procesos eran poco selectivos y sus rendimientos eran bajos, mientras que el proceso de BASF permitía tener selectividades hacia ácido acético mayores al 90\%. No obstante, una limitación del proceso de BASF es que requería de altas presiones (500$700 \mathrm{~atm}$ ), por lo que existía interés en encontrar catalizadores que pudieran llevar a cabo la reacción a condiciones más favorables. A finales de los años sesenta del siglo XX, Monsanto desarrolló un proceso de carbonilación de metanol que resolvía este problema al utilizar $\mathrm{Rh}(\mathrm{CO})_{2} \mathrm{I}_{2}$ - como catalizador. Nuevamente, se requería yodometano como cocatalizador, pero la alta reactividad de los complejos de Rh permitía llevar a cabo la carbonilación a presiones entre 30 y $40 \mathrm{~atm}$. Además, la selectividad hacia ácido acético era también mayor que la del proceso de BASF (>99\%) (Paulik y Roth, 1968). Rápidamente, el proceso de Monsanto se convirtió en un referente para la producción de ácido acético.

Investigación desarrollada por la misma empresa y por diversos grupos de investigación (Paulik y Roth, 1968; Foster, 1979) permitió establecer el ciclo catalítico de la carbonilación de metanol en presencia de $\mathrm{Rh}(\mathrm{CO})_{2} \mathrm{I}_{2}$-(figura 3). En el mecanismo se propone que el complejo experimenta la adición oxidativa de yodometano para formar un complejo de 18 electrones que posteriormente sufre la inserción de $\mathrm{CO}$ para formar el grupo acetil enlazado al rodio. El ciclo principal termina con la producción de $\mathrm{CH}_{3} \mathrm{COI}$ y la regeneración de $\mathrm{Rh}(\mathrm{CO})_{2} \mathrm{I}_{2}$ - El ácido acético es entonces obtenido a partir de la hidrólisis de $\mathrm{CH}_{3} \mathrm{COI}$, mientras que el cocatalizador de yodometano se recupera a partir de la reacción entre el metanol y el yoduro de hidrógeno (figura 3, ciclo inferior izquierdo).

Existe suficiente evidencia espectroscópica que respalda el ciclo mostrado en la figura 2, de modo que la reacción es comprendida a cierto detalle (Foster, 1979). Entre otras cosas, el mecanismo indica la participación de complejos $\mathrm{d}^{6} \mathrm{y} \mathrm{d}^{8}$ de $\mathrm{Rh}^{\mathrm{I}}$ y $\mathrm{Rh}^{\mathrm{III}}$, respectivamente. Este descubrimiento permitió buscar complejos isolectrónicos de otros metales que (en principio) se esperaría que también fueran catalíticamente activos para la reacción. Así, en 1968 se descubrió que complejos de $\operatorname{Ir}^{\mathrm{I}}$ e $\mathrm{Ir}^{\mathrm{III}}$ también funcionaban satisfactoriamente para la reacción (Paulik y Roth, 1968). Estos estudios sirvieron como base para que se desarrollara el proceso Cativa ${ }^{\mathrm{TM}}$ por la empresa BP Chemicals en 1995 (Haynes et al., 2004).

Los procesos descritos anteriormente tienen la desventaja de ser homogéneos, lo que dificulta la separación y recuperación del catalizador. Por ello, desde que el proceso de BASF fue propuesto, existieron intentos de preparar catalizadores sólidos que fueran eficientes para la carbonilación de metanol. A lo largo del tiempo se probaron diversas combinaciones de metales (casi siempre Rh, Co e Ir, en intentos por imitar los procesos instalados industrial- 
Figura 3. Ciclo catalítico propuesto para la carbonilación de metanol en presencia del catalizador de Monsanto.

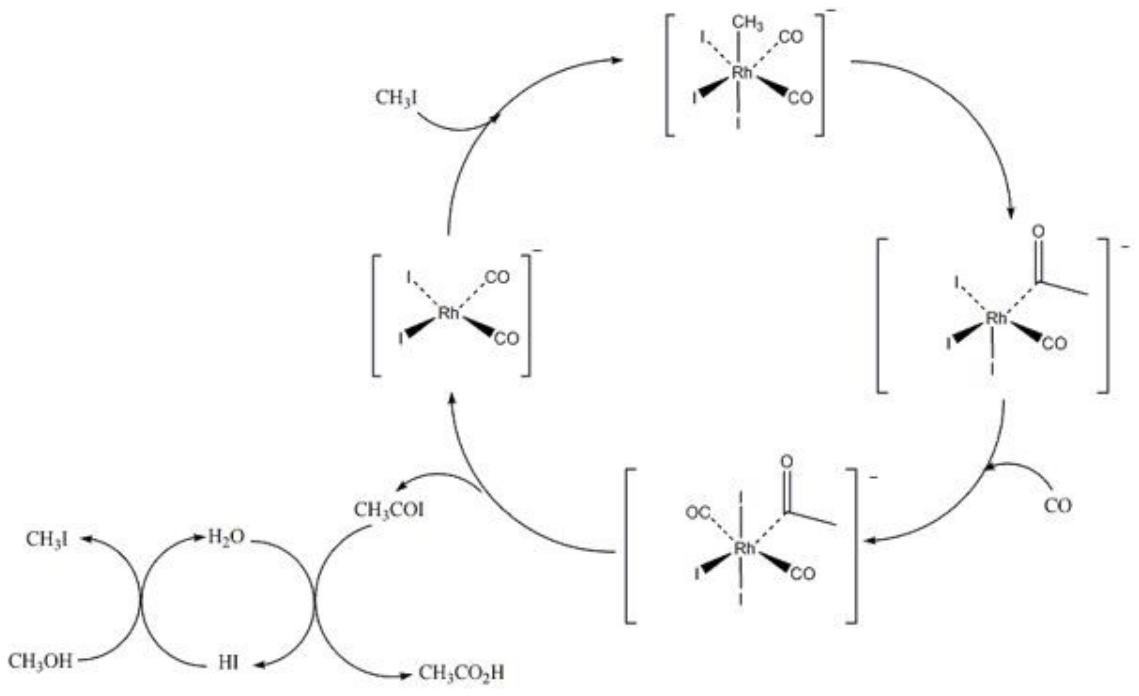

Fuente: Elaboración del autor.

mente) soportados en óxidos metálicos, pero los catalizadores normalmente eran menos activos, selectivos o estables que sus contrapartes usadas en procesos homogéneos. Uno de los principales problemas era que el metal no se anclaba de manera suficientemente estable sobre el soporte, por lo que normalmente lixiviaba en condiciones de reacción. El grupo de Cole-Hamilton (De Blasio et al., 1998) reportó la carbonilación de metanol catalizada por rodio soportado en PVP y en poliestireno entrecruzado modificado con ligantes de fosfinas. Los autores llevaron a cabo la reacción alimentando los reactivos en fase gas y demostraron que, en esas condiciones, el rodio permanecía en la superficie de los soportes. Más aún, la actividad catalítica y selectividad del material era comparable a la observada en los procesos heterogéneos. La idea detrás del empleo de los soportes propuestos por Cole-Hamilton y su grupo era la posibilidad de formar interacciones de pares iónicos suficientemente fuertes entre sitios de pirrolidona cuaternaria y aniones de $\mathrm{Rh}(\mathrm{CO})_{2} \mathrm{I}_{2}$ - que se intentaba preparar desde la síntesis de los materiales. Debe enfatizarse que los autores partieron del conocimiento de que los complejos de $\mathrm{Rh}(\mathrm{CO})_{2} \mathrm{I}_{2}$ - deberían funcionar como catalizadores, según lo habían demostrado las investigaciones realizadas sobre el proceso de Monsanto. La industria tomó nota de los descubrimientos y avances en la estabilización de complejos de Rh en soportes, de modo que en 1998, Chiyoda y UOP desarrollaron el proceso Acetica ${ }^{\mathrm{TM}}$, en el que se emplean complejos de Rh soportados en polivinilpiridina como catalizador para producir ácido acético a partir de la carbonilación de metanol. Se ha reportado que el catalizador es estable a relativamente altas presiones y tem- 
peraturas, al tiempo que permite obtener rendimientos similares a los reportados para sus contrapartes de procesos homogéneos. En 2002, la empresa Guizhou Crystal Organic Chemical Group negoció una licencia para utilizar el proceso Acetica ${ }^{\mathrm{TM}}$ en una planta que instaló en China con una capacidad de producción de 36,000 toneladas anuales de ácido acético. En la figura 4 se incluye una línea del tiempo que muestra los avances más significativos en la carbonilación del metanol, desde el descubrimiento de los complejos usados para los procesos homogéneos hasta la puesta en marcha del proceso heterogéneo. Este ejemplo es un caso de éxito en el que la comprensión a nivel fundamental del modo en que un metal (en este caso $\mathrm{Rh}$ ) cataliza una reacción en solución sirvió como base para diseñar un catalizador sólido.

\section{Reformado catalítico de nafta del petróleo}

Quizás uno de los ejemplos más notables del desarrollo de un catalizador sólido a partir de ideas fundamentales sobre su funcionamiento se encuentra en el proceso de reformado de nafta. En la industria del petróleo, el término reformado de nafta se refiere al proceso mediante el que la estructura molecular de los compuestos presentes en las naftas se altera con la intención de aumentar su octanaje para su uso como combustible en motores de combustión interna. En las gasolinas, los cuatro tipos de compuestos normalmente encontrados incluyen parafinas, olefinas, naftalenos y aromáticos. Las parafinas lineales son los compuestos con menor octanaje. A medida que las parafinas son más ramificadas, los valores de índice de octano aumentan. Típicamente, las olefinas tienen mayor octanaje que sus parafinas correspondientes, al igual que las cicloparafinas (o naftenos). Finalmente, los compuestos aromáticos son los que ofrecen el mayor valor de índice de octano. No existe una regla precisa para establecer el valor del octanaje para cada compuesto y asociarlo con su estructura, pero las tendencias que se mencionaron anteriormente suelen cumplirse en las gasolinas.

Figura 4. Línea del tiempo de desarrollo de catalizadores para la carbonilación de metanol. Inicia con los procesos homogéneos y concluye con el proceso heterogéneo inspirado en ellos.

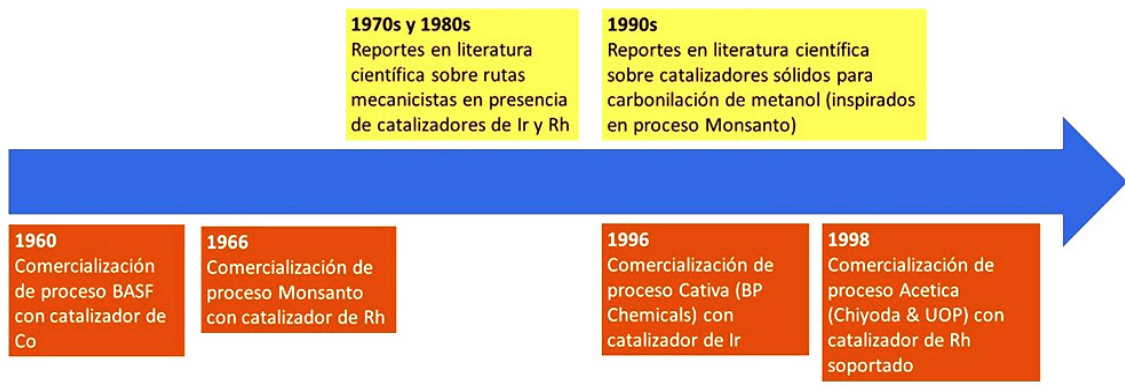

Fuente: Elaboración del autor. 
El primer proceso que se usó en la industria petroquímica para modificar la composición de naftas con la intención de aumentar su octanaje fue el reformado térmico. Este proceso se comenzó a emplear aproximadamente en 1930 y consistía en tratar las naftas a temperaturas por encima de los 500 ${ }^{\circ} \mathrm{C}$ y presiones entre 30 y $60 \mathrm{~atm}$. A esas condiciones, algunas parafinas se convertían en olefinas, mientras que las parafinas de mayor peso molecular se fragmentaban (i.e., experimentaban cracking) para producir parafinas de menor peso molecular. Con ello, la concentración de los compuestos aromáticos aumentaba en la mezcla líquida. Así pues, el proceso permitía aumentar el octanaje de las gasolinas, pero a niveles aún muy lejanos a los que actualmente serían aceptables.

Los problemas principales del reformado térmico eran que no se creaban nuevos compuestos aromáticos y además había una baja eficiencia debido al excesivo cracking de parafinas. En la década de los cuarenta, investigadores de la empresa Universal Oil Products (UOP) abordaron esos problemas proponiendo un proceso de reformado catalítico (Sterba y Haensel, 1976). El objetivo era encontrar un catalizador que pudiera aumentar el octanaje sin las limitaciones del reformado térmico. En lugar de aproximarse al problema mediante la prueba y error, decidieron primero definir las características que (al menos teóricamente) debería tener el catalizador para funcionar. Así, los investigadores propusieron que un buen catalizador para aumentar el octanaje debería ser capaz de llevar a cabo tanto reacciones hidrogenación como de deshidrogenación. Como los productos que otorgarían alto octanaje al producto serían alquenos altamente ramificados, el catalizador debería ser también bueno para llevar a cabo reacciones de isomerización de alquenos. En esa época ya se tenía conocimiento de que la isomerización de alquenos en solución era catalizada por ácidos. Por lo tanto, los investigadores determinaron que el catalizador sólido debería además tener suficientes propiedades ácidas.

A partir de esas ideas, los investigadores de UOP propusieron que el catalizador más apropiado para el proceso debería contener nanopartículas de un metal que se distribuyeran uniformemente sobre un soporte poroso. La idea era que el metal debería catalizar las reacciones de deshidrogenación de parafinas, de modo que las olefinas resultantes pudieran migrar con relativa facilidad hacia el soporte, donde se esperaba que los sitios ácidos de este favorecieran su isomerización. Algunos de los productos ramificados podrían entonces volver a ser hidrogenados en la superficie del metal. El proceso, en forma global, debería dar lugar a altos octanajes de la mezcla resultante. Con este razonamiento, se propuso utilizar nanopartículas de platino soportadas en óxido de aluminio como catalizador para el proceso. Se puede considerar que el catalizador es bifuncional, pues el metal tiene la función de producir olefinas a partir de parafinas, mientras que el soporte tiene la función de formar olefinas ramificadas.

Desde el descubrimiento de los investigadores de UOP han surgido mejoras al proceso y al catalizador original. Debe decirse que muchas de esas modificaciones han sido realizadas usando una aproximación de prueba y error. 
Sin embargo, las pruebas realizadas parten del conocimiento que fue desarrollado a lo largo del tiempo y que permiten generar hipótesis sobre los soportes y metales que tendrían potencial de funcionar adecuadamente en la reacción. Es decir, no se trata de un extremo de prueba y error. Aunque, tampoco se ha llegado al diseño puro, en el que se pueda predecir con exactitud qué material será efectivamente inmejorable y simplemente prepararlo. Así pues, el reformado catalítico es un caso que demuestra que el desarrollo de catalizadores (de manera práctica) utiliza elementos de diseño y de métodos de prueba y error. Esto no se debe a la falta de una comprensión significativa del problema, sino a las dificultades asociadas para preparar catalizadores que contengan exclusivamente las especies superficiales que se requieren para el proceso. Este ejemplo resalta que la lógica usada por quienes desarrollaron el catalizador está basada en una comprensión fundamental sobre el modo en que se esperaba que el catalizador funcionara. Esta lógica (y la estrategia que de ella se deriva) es más parecida al diseño que a la prueba y error.

\section{Catalizadores sólidos imitando el funcionamiento de enzimas: reacción de Diels-Alder catalizada por zeolitas}

Las enzimas son macromoléculas que funcionan como catalizadores biológicos y tienen estructuras tridimensionales sumamente complicadas. Las enzimas contienen cientos de aminoácidos unidos entre sí a través de enlaces peptídicos en arreglos espaciales sorprendentemente complejos. Estos compuestos son esenciales para la vida, pues regulan múltiples procesos metabólicos. En la catálisis enzimática, los sustratos (reactivos) se enlazan a las enzimas en sitios específicos, lo que hace que las enzimas sean catalizadores altamente selectivos. Los enlaces entre las enzimas y los sustratos pueden ocurrir a través de coordinación con metales (en el caso de metaloenzimas), pares iónicos, coordinación en sitios ácido-base, o a través de puentes de hidrógeno. Debido a la alta selectividad y actividad catalítica de las enzimas, han existido intentos por imitar de manera artificial su funcionamiento. Así, se han preparado una variedad de materiales, que incluyen ciclodextrinas, porfirinas, polímeros, dendrímeros y zeolitas, entre otros (Breslow,1995; Darbre y Reymond, 2006; Dessau,1986). Algunos de estos materiales han mostrado actividad y selectividad prometedoras, lo que ofrece oportunidades interesantes hacia el diseño de catalizadores con propiedades específicas. No obstante, en la mayoría de los casos, aunque los materiales tienen arquitecturas de poro definidas, estas no necesariamente se parecen a la que tienen las enzimas. Por ello, los paralelismos entre esos materiales y las enzimas son limitados. Un ejemplo reciente en el que verdaderamente se logró diseñar un material con estructuras parecidas a las de sitios activos de enzimas es el trabajo del grupo de Corma y col (Gallego et al., 2019), quienes estudiaron la reacción de Diels Alder (DA) utilizando como catalizador una zeolita (Si-BEC) diseñada específicamente para tal proceso y que imita el funcionamiento de enzimas. 
La reacción de DA es una cicloadición entre un dieno conjugado y un alqueno (o dienófilo). El proceso es importante porque permite formar dos nuevos enlaces $C-C$ y hasta cuatro centros estereogénicos en un solo paso. El mecanismo de la reacción ha sido ampliamente estudiado en procesos homogéneos, y se ha propuesto que en él participa un estado de transición (ET) que tiene una estructura altamente ordenada y similar a la del producto.

Se ha encontrado que existen algunas enzimas que catalizan reacciones de cicloadición similares a la de DA (Oikawa y Tokiwano, 2004). En esos casos, se ha propuesto que los sitios activos de la enzima tienen una geometría tridimensional similar a la del ET de la reacción que catalizan. Partiendo de esa propuesta, el grupo de Corma sugirió la posibilidad de sintetizar zeolitas que tuvieran cavidades con geometría tridimensional similar al ET que se esperaría en una reacción de DA. Específicamente, estudiaron la reacción entre 1,3-ciclohexadieno y $\mathrm{N}$-metilmaleimida. El reto principal consistía entonces en lograr sintetizar un material con una morfología específica. Para ello, utilizaron una estrategia que habían desarrollado con éxito anteriormente, en la que se emplea un agente orgánico director de estructura que funciona como una plantilla para crear cavidades de la zeolita. En este caso, el agente utilizado tenía una estructura similar a la que se esperaba tendría el ET de la reacción. El razonamiento del grupo de Corma y colaboradores (Gallego et al., 2019) era que las cavidades estabilizaran al ET, y de ese modo la reacción de DA ocurriría de manera selectiva. Su hipótesis fue comprobada cuando probaron el material sintetizado como catalizador y encontraron velocidades de reacción aproximadamente tres veces mayores a las reportadas en presencia de otros catalizadores sólidos convencionales. Este ejemplo es quizás lo más cercano a uno diseño ab initio de catalizadores. Puede verse que el éxito de la aproximación depende no únicamente del nivel de comprensión sobre el mecanismo de la reacción, sino que además se requiere de la capacidad técnica suficiente para sintetizar el material que propone el diseño.

\section{Pragmatismo en el diseño de catalizadores sólidos: el presente y un futuro posible}

El ejemplo discutido anteriormente sugiere que uno de los retos principales a los que se enfrenta el desarrollo de catalizadores basado en el diseño es contar con herramientas y estrategias suficientemente desarrolladas para sintetizar materiales con estructuras específicas. Además, se requiere del uso complementario de técnicas de caracterización para verificar la uniformidad de las superficies de los materiales. Afortunadamente, los avances actuales en la resolución espacial y de tiempo en técnicas de espectroscopía y microscopía hacen que sea posible estudiar estructuras superficiales a gran detalle.

Lo anterior sugiere que cada vez más, los grupos de investigación de catálisis heterogénea deberán desarrollar protocolos de preparación de mate- 
riales en los que se tenga un mayor control químico durante la síntesis. Esto implica que la descripción de la superficie de los catalizadores deberá realizarse en términos rigurosos desde el punto de vista químico. Ya no será suficiente utilizar variables estructurales convencionales, como tamaños promedio de partículas soportadas, mediciones indirectas del estado de oxidación del metal, o distribuciones de tamaños de poro basadas en mediciones de área superficial. Será necesario, además, describir la superficie y los sitios activos en términos de los enlaces químicos que se forman entre sus componentes. En ese sentido, los investigadores que realizan trabajo experimental se verán beneficiados cada vez más de los avances notables que han surgido en química computacional, pues esta herramienta podrá servir como una guía para los protocolos sintéticos. Lo anterior no significa que dejará de usarse la prueba y error para desarrollar catalizadores. En realidad, se requerirá de una aproximación pragmática en la que elementos del diseño y de la prueba y error se combinen para abordar problemas de catálisis. No debe perderse de vista que existe una amplia variedad de tipos de catalizadores sólidos que se usan para diversas aplicaciones. En algunos casos, elementos del diseño podrán ser de utilidad, pero esto no necesariamente se cumplirá en todos los casos. Hay catalizadores que se usan a nivel industrial que funcionan considerablemente bien, pero que son extremadamente complejos en su estructura. Para su mejoramiento, una aproximación basada en el diseño podría representar retos enormes. En esos casos, el uso de prueba y error parecería ser más conveniente y adecuado.

Así pues, existe un aparente dilema entre el uso de la prueba y error y el diseño. Situaciones similares se presentan en otras ramas de la ciencia, y tal vez la analogía más cercana se encuentra en el desarrollo de fármacos, en donde una estrategia común para generar nuevos medicamentos o vacunas consiste en el llamado high-throughput screening (a veces traducido como cribado de alta capacidad). La aproximación consiste aumentar la cantidad de experimentos que pueden hacerse para probar compuestos candidatos como fármacos. Ello requiere utilizar diversas herramientas, que incluyen desde la robótica para sintetizar compuestos, hasta el software de procesamiento y análisis de grandes cantidades de datos, entre otras. De este modo se pueden comparar miles de compuestos e identificar rasgos o características (recuérdese le definición etimológica del diseño que se explicó al inicio de este artículo) particulares que hacen que algunos de ellos funcionen mejor que otros para la prevención o el tratamiento de enfermedades. De este modo, los resultados son útiles al proporcionar información que sirve como punto de partida para el diseño de fármacos. Esta estrategia también ha sido discutida ampliamente en la literatura para desarrollar catalizadores sólidos en los últimos años (Corma, 2016; Ertl, 2018). Recientemente, el grupo de Lauterbach reportó (Williams et al., 2020) el uso de esta aproximación en combinación con métodos de machine learning (ML, a veces traducido como aprendizaje automatizado de máquinas). La premisa detrás de los métodos de ML es utilizar inteligencia artificial para que un 
programa de análisis de datos (o un robot que fabrique algún material, por ejemplo) determine patrones sin haber sido programado originalmente de manera explícita para reconocerlos. Con este enfoque, Lauterbach y colaboradores desarrollaron una metodología en la que incorporaron al método de ML características químicas como la composición, morfología y propiedades electrónicas de catalizadores usados en la síntesis de amoníaco. Al probar su método, fueron capaces de llegar a una formulación de un catalizador novedoso de $\mathrm{RuYK} / \mathrm{Al}_{2} \mathrm{O}_{3}$ con menor costo y mayor actividad que los de catalizadores reportados en el pasado para la síntesis de amoníaco. Los autores resaltan que la incorporación de métodos de ML dio lugar a una disminución del número de datos experimentales necesarios para encontrar patrones sobre el funcionamiento del catalizador y predecir la composición de un mejor material.

El ejemplo anterior ilustra el modo en que pueden combinarse la prueba y error, el diseño y los avances tecnológicos para apoyar el desarrollo de catalizadores. Al final, preparar catalizadores (independientemente de la estrategia que se haya elegido para hacerlo) seguirá teniendo una dosis de arte y de habilidades que permitan a los investigadores llevar conceptos aparentemente abstractos a protocolos de síntesis y caracterización que sean realistas en función de los avances tecnológicos. Pero más allá de eso, los ejemplos que se discuten en este artículo pretenden mostrar que el ingenio, la intuición y la inventiva serán siempre esenciales para el avance en el estudio de catalizadores.

\section{Referencias}

Argo, A. M.; Odzak, J. F.; Lai, F. S.; Gates, B. C. (2002). Observation of ligand effects during alkene hydrogenation catalysed by supported metal clusters. Nature, 415: 623. https://doi.org/10.1038/415623a

Bond, G. C. (1991). Supported metal catalysts: some unsolved problems. Chemical Society Reviews, 20:441. https://doi.org/10.1039/CS9912000441

Breslow, R. (1995). Biomimetic chemistry and artificial enzymes: catalysis by design. Accounts of Chemical Research, 28:146. https://doi.org/10.1021/ar00051a008

Brown, J. M; Evans, P. L.; Lucy, A. R. (1987). Stereochemistry of intermediates in homogeneous hydrogenation catalysed by tristriphenylphosphinerhodium chloride, employing nuclear magnetic resonance magnetisation transfer. Journal of the Chemical Society, Perkin Transactions, 2(1): 1589. https://doi.org/10.1039/ P29870001589

Burch, R.; Breen. J. P.; Meunier, F. C. (2002). A review of the selective reduction of $\mathrm{NOx}$ with hydrocarbons under lean-burn conditions with non-zeolitic oxide and platinum group metal catalysts. Applied Catalysis B: Environmental, 39(4): 283. https://doi.org/10.1016/S0926-3373(02)00118-2

Corma, A. (2016). Heterogeneous catalysis: understanding for designing, and designing for applications. Angewandte Chemie. International Edition, 55(21): 6112. https://doi.org/10.1002/anie.201601231 
Corma, A.; Corresa, E.; Mathieu, Y.; Sauvanaud, L.; Al-Bogami, S.; Al-Ghrami, M. S.; Bourane, A. (2017). Crude oil to chemicals: light olefins from crude oil. Catalysis Science \& Technology, 7: 12. https://doi.org/10.1039/C6CY01886F

Corma, A.; García, H.; Llabrés i Xamena, F. X. (2010). Engineering metal organic frameworks for heterogeneous catalysis. Chemical Reviews, 110(8):4606. https://doi. org/10.1021/cr9003924

Daniel, C.; Koga, N.; Han, J.; Fu, X. Y.; Morokuma, K. (1988). Ab initio MO study of the full catalytic cycle of olefin hydrogenation by the Wilkinson catalyst $\mathrm{RhCl}\left(\mathrm{PR}_{3}\right)_{3}$. Journal of the American Chemical Society, 110(12): 3773. https:// doi.org/10.1021/ja00220a010

Darbre, T.; Reymond, J.-L. (2006). Peptide dendrimers as artificial enzymes, receptors, and drug-delivery agents. Accounts of Chemical Research, 39(12): 925. DOI: 10.1021/ar050203y

De Blasio, N.; Wright, M. R.; Tempesti, E.; Mazzocchia, C.; Cole-Hamilton, D. J. (1998). The relative importance of heterogeneous and homogeneous methanol carbonylation using supported rhodium catalysts in the liquid phase. Journal of Organometallic Chemistry, 551(1-2):229. https://doi.org/10.1016/S0022-328X(97)00438-5

De María, R.; Díaz, I.; Rodríguez, M.; Sáiz, A. (2013). Industrial methanol from syngas: kinetic study and process simulation. International Journal of Chemical Reactor Engineering, 11(1): 469. DOI: 10.1515/ijcre-2013-0061

Dessau, R. M. (1986). Catalysis of Diels-Alder reactions by zeolites. Journal of the Chemical Society, Chemical Communications, 1: 1167. https://doi.org/10.1039/ C39860001167

Ertl, G. (2018). Heterogeneous catalysis: where are we? Catalysis in Chemistry and Biology, 1: 61. https://doi.org/10.1142/9789813237179_0012

Fierro-González, J. C.; Bhirud, V. A.; Gates, B. C. (2005). A highly active catalyst for CO oxidation at $298 \mathrm{~K}$ : mononuclear $\mathrm{Au}^{\mathrm{III}}$ complexes anchored to $\mathrm{La}_{2} \mathrm{O}_{3}$ nanoparticles. Chemical Communications, 1(42): 5275. https://doi.org/10.1039/B509629D

Fierro-González, J. C.; Gates, B. C. (2004). Mononuclear $\mathrm{Au}^{\mathrm{III}}$ and $\mathrm{Au}^{\mathrm{I}}$ complexes bonded to zeolite NaY: catalysts for CO oxidation at $298 \mathrm{~K}$. Journal of Physical Chemistry B, 108(44): 16999. https://doi.org/10.1021/jp046171y

Fihri, A.; Bouhrara, M.; Nekoueishahraki, B.; Basset, J.-M.; Polshettiwar, V. (2011). Nanocatalysts for Suzuki cross-coupling reactions. Chemical Society Reviews, 40(10): 5181. https://doi.org/10.1039/C1CS15079K

Foster, D. (1979). Mechanistic pathways in the catalytic carbonylation of methanol by rhodium and iridium complexes. Advances in Organometallic Chemistry, 17:c255. https://doi.org/10.1016/S0065-3055(08)60325-1

Gallego, E. M.; Paris, C.; Cantín, A.; Moliner, M.; Corma, A. (2019). Conceptual similarities between zeolites and artificial enzymes. Chemical Science, 10: 8009. https://doi.org/10.1039/C9SC02477H

Gates, B. C. (1995). Supported metal clusters: synthesis, structure, and catalysis. 95(3): 511. https://doi.org/10.1021/cr00035a003

Goodman, D. W. (2008). Precious little catalyst. Nature, 454: 948. https://doi. $\operatorname{org} / 10.1038 / 454948$ a 
Mundo Nano | ARTículos DE REVISIóN | www.mundonano.unam.mx

14(26), 1e-21e, enero-junio 2021 | https:/ / doi.org/10.22201/ceiich.24485691e.2021.26.69645

Juan C. Fierro-Gonzalez

Hamada, H. (1994). Selective reduction of NO by hydrocarbons and oxygenated hydrocarbons over metal oxide catalysts. Catalysis Today, 22(1): 21. https://doi. org/10.1016/0920-5861(94)80090-1

Haynes A. (2010). Catalytic methanol carbonylation. Advances in Catalysis, 53: 1. https://doi.org/10.1016/S0360-0564(10)53001-3

Haynes, A.; Maitlis, P. M.; Morris, G. E.; Sunley, G. J,; Adams, H.; Badger, P. W.; Bowers, C. M.; Cook, D. B.; Elliott, P. I. P.; Ghaffar, T.; Green, H.; Griffin, T. R.; Payne, M.; Pearson, J. M.; Taylor, M. J.; Vickers, P. W.; Watt, R. J. (2004). Promotion of iridium-catalyzed methanol carbonylation: mechanistic studies of the Cativa process. Journal of the American Chemical Society, 126(9): 2847. https://doi. org/10.1021/ja039464y

$\mathrm{He}, \mathrm{H}$.; Yu, Y. (2005). Selective catalytic reduction of $\mathrm{NOx}$ over $\mathrm{Ag} / \mathrm{Al}_{2} \mathrm{O}_{3}$ catalyst: from reaction mechanism to diesel engine test. Catalysis Today, 100(1-2): 37. https://doi.org/10.1016/j.cattod.2004.11.006

Jentoft, R. E.; Tsapatsis, M.; Davis, M. E.; Gates, B. C. (1998). Platinum clusters supported in zeolite LTL: influence of catalyst morphology on performance in nHexane eforming. Journal of Catalysis, 179: 565. https://doi.org/10.1006/ jcat.1998.2229

Jira, R. (2009). Acetaldehyde from ethylene-a retrospective on the discovery of the Wacker process. Angewandte Chemie, International Edition, 48(48): 9034. https:// doi.org/10.1002/anie.200903992

Liu, W., Rodriguez, P., Borchardt, L.; Foelske, A.; Yuan, J.; Herrmann, A. K.; Geiger, D.; Zheng, Z.; Kaskel, S.; Gaponik, N.; Kotz, R.; Schmidt, T. J.; Eychmuller, A. (2013). Bimetallic aerogels: high-performance electrocatalysts for the oxygen reduction reaction. Angewandte Chemie International Edition, 52: 9849. https:// doi.org/10.1002/anie.201303109

McNaught, A. D. y Wilkinson, A. (1997) Compendium of Chemical Terminology, 2a ed. (the "Gold Book”). Oxford: Blackwell Scientific Publications. IUPAC. [ISBN 847738-955-1].

Minami, T.; Shimokawa, K.; Hamato, K.; Shiroto, Y.; Yoneda, N. (1994). Supported rhodium catalyst, method of preparing same and process of producing acetic acid by methanol carbonylation using same. US Patent 5364963A.

Oikawa, H.; Tokiwano, T. (2004). Enzymatic catalysis of the Diels-Alder reaction in the biosynthesis of natural products. Natural Products Reports, 21: 321. https://doi.org/10.1039/B305068H

Osbom, J. A.; Jardine, F. H.; Young, J. F.; Wilkinson, G. The preparation and properties of tris(triphenylphosphine)halogenorhodium(I) and some reactions thereof including catalytic homogeneous hydrogenation of olefins and acetylenes and their derivatives. Journal of the Chemical Society A: Inorganic, Physical and Theoretical, 171: 1. https://doi.org/10.1039/J19660001711

Paulik, F. E.; Roth, J. F. (1968). Novel catalysts for the low-pressure carbonylation of methanol to acetic acid. Chemical Communications, 1: 1578a. https://doi. org/10.1039/C1968001578A

Primo, A.; Garcia, H. (2014). Zeolites as catalysts in oil refining. Chemical Society 
Reviews, 43(22): 7548. https://doi.org/10.1039/c3cs60394f

Pruett, R. L.; Smith, J. A. (1967). Hydroformylation process. US Patents, US3527809A.

Reppe, W.; Friederich, H.; von Kutepow, N.; Morsch, W. (1956). Process for the production of aliphatic oxygen compounds by carbonylation of alcohols, ethers, and esters. US Patents, US2729651A.

Soulivong, D.; Norsic, S.; Taoufik, M.; Coperet, C.; Thivolle-Cazat, J.; Chakka, S.; Basset, J.-M. (2008). Non-oxidative coupling reaction of methane to ethane and hydrogen catalyzed by the silica-supported tantalum hydride: $(\equiv \mathrm{SiO})_{2} \mathrm{Ta}-\mathrm{H}$. Journal of the American Chemical Society, 130(15): 5044. https://doi.org/10.1021/ ja800863x

Sterba, M. J.; Haensel, V. (1976). Catalytic reforming. Industrial \& Engineering Chemistry Product Research and Development, 15: 2.

Thomas, J. M. (1999). Design, synthesis, and in situ characterization of new solid catalysts. Angewandte Chemie International Edition, 38(24): 3588. https://doi. org/10.1002/(SICI)15213773(19991216)38:24<3588::AIDANIE3588>3.0.CO;2-4

Williams, T.; McCullough, K.; Lauterbach, J. A. (2020). Enabling catalyst discovery through machine learning and high-throughput experimentation. Chemistry of Materials, 32(1): 157. https://doi.org/10.1021/acs.chemmater.9b03043

Yang, D.; Odoh, S. O.; Wang, T. C.; Farha, O. K.; Hupp, J. T.; Cramer, C. J.; Gagliardi, L.; Gates, B. C. (2015). Metal-organic framework nodes as nearly ideal supports for molecular catalysts: NU-1000- and UiO-66-supported iridium complexes. Journal of the American Chemical Society, 137(23):7391. https://doi. org/10.1021/jacs.5b02956 